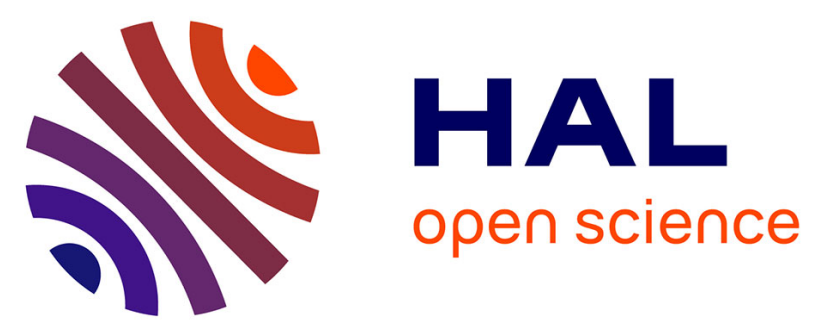

\title{
Symmetry aspects of spin filtering in molecular junctions: Hybridization and quantum interference effects
}

Dongzhe Li, Rajdeep Banerjee, Sourav Mondal, Ivan Maliyov, Mariya

Romanova, Yannick J Dappe, Alexander Smogunov

\section{To cite this version:}

Dongzhe Li, Rajdeep Banerjee, Sourav Mondal, Ivan Maliyov, Mariya Romanova, et al.. Symmetry aspects of spin filtering in molecular junctions: Hybridization and quantum interference effects. Physical Review B: Condensed Matter and Materials Physics (1998-2015), 2019, 99 (11), 10.1103/PhysRevB.99.115403 . hal-02336475

\section{HAL Id: hal-02336475 \\ https://hal.science/hal-02336475}

Submitted on 28 Oct 2019

HAL is a multi-disciplinary open access archive for the deposit and dissemination of scientific research documents, whether they are published or not. The documents may come from teaching and research institutions in France or abroad, or from public or private research centers.
L'archive ouverte pluridisciplinaire HAL, est destinée au dépôt et à la diffusion de documents scientifiques de niveau recherche, publiés ou non, émanant des établissements d'enseignement et de recherche français ou étrangers, des laboratoires publics ou privés. 


\title{
Symmetry aspects of spin filtering in molecular junctions: Hybridization and quantum interference effects
}

\author{
Dongzhe Li, ${ }^{1, *}$ Rajdeep Banerjee, ${ }^{2}$ Sourav Mondal, ${ }^{2}$ Ivan Maliyov, ${ }^{3}$ Mariya Romanova, ${ }^{4}$ Yannick J. Dappe, ${ }^{5}$ \\ and Alexander Smogunov 5 \\ ${ }^{1}$ Department of Physics, University of Konstanz, 78457 Konstanz, Germany \\ ${ }^{2}$ Theoretical Sciences Unit and School of Advanced Materials, Jawaharlal Nehru Centre for Advanced Scientific Research, \\ Jakkur, Bangalore 560064, India \\ ${ }^{3}$ DEN, Service de Recherches en Métallurgie Physique, CEA, Université Paris-Saclay, 91191 Gif-sur-Yvette Cedex, France \\ ${ }^{4}$ Laboratoire des Solides Irradiés, Ecole Polytechnique, CEA-DRF-IRAMIS, CNRS UMR 7642, 91120 Palaiseau, France \\ ${ }^{5}$ SPEC, CEA, CNRS, Université Paris-Saclay, CEA Saclay, 91191 Gif-sur-Yvette Cedex, France
}

(Received 21 November 2018; revised manuscript received 12 February 2019; published 5 March 2019)

\begin{abstract}
Control and manipulation of electric current and, especially, its degree of spin polarization (spin filtering) across single molecules are currently of great interest in the field of molecular spintronics. We explore one possible strategy based on the modification of nanojunction symmetry which can be realized, for example, by a mechanical strain. Such modification can activate new molecular orbitals which were inactive before due to their orbital mismatch with the electrode's conduction states. This can result in several important consequences such as (i) quantum interference effects appearing as Fano-like features in electron transmission and (ii) the change in molecular level hybridization with the electrode's states. We argue that the symmetry change can affect very differently two majority- and minority-spin conductances and thus alter significantly the resulting spin-filtering ratio as the junction symmetry is modified. We illustrate the idea for two basic molecular junctions: $\mathrm{Ni}$ /benzene/Ni (perpendicular vs tilted orientations) and $\mathrm{Ni} / \mathrm{Si}$ chain/Ni (zigzag vs linear chains). In both cases, one highest occupied molecular orbital (HOMO) and one lowest unoccupied molecular orbital (LUMO) (out of HOMO and LUMO doublets) are important. In particular, their destructive interference with other orbitals leads to dramatic suppression of majority-spin conductance in low-symmetry configurations. For a minority-spin channel, on the contrary, the conductance is strongly enhanced when the symmetry is lowered due to an increase in hybridization strength. We believe that our results may offer a potential route for creating molecular devices with a large on-off ratio of spin polarization via quantum interference effects.
\end{abstract}

DOI: 10.1103/PhysRevB.99.115403

\section{INTRODUCTION}

Theoretical understanding and experimental realization of electron transport through single-molecule junctions with ferromagnetic electrodes, especially with the goal to control and manipulate its degree of spin polarization, are currently of great importance in the field of molecular spintronics [1,2], a rapidly developing branch of nanoelectronics. Among many properties of interest is the spin-filtering ratio [3] (or spin polarization, SP), which measures the degree of spin polarization of the electronic conductance. High values of SP are usually accompanied by large magnetoresistance ratios (MRRs) measuring the difference in conductance between parallel and antiparallel magnetic orientations of two ferromagnetic electrodes. It is also a great challenge to suggest possible systems and measurements where solid symmetry arguments and quantum effects such as destructive (or constructive) interference could play an important role in spin polarization and could be tested experimentally.

*dongzhe.li@uni-konstanz.de
At the single-molecule scale, more than $100 \%$ of MRRs were predicted theoretically for $\mathrm{Fe} / \mathrm{C}_{60} / \mathrm{Fe}$ magnetic junctions [4], and moderate values of MRRs up to $60 \%$ were recently reported [5,6]. These effects were attributed to the spin-dependent hybridization occurring at the ferromagnet/molecule interface. Also, large spin polarizations of different organic molecules due to hybridization with magnetic substrates were reported [7-10]. This can result in large tunneling magnetoresistance, as shown, for example, for $\mathrm{C}_{60}$ molecules on $\mathrm{Cr}(001)$ terraces [9]. Regarding atomic nanocontacts, giant magnetoresistance values of about $70 \%$ were reported recently for $\mathrm{Co} / \mathrm{Au} / \mathrm{Co}$ metallic junctions [11] as a result of strong perturbation of $s$ states at Au contact atoms, and very large spin polarizations of the current were found in half-metallic NiO monatomic junctions [12]. Rather detailed analysis done by Bagrets and coworkers [13] for short (three atoms long) chains of various species joining two Co electrodes also revealed, in some cases, MRRs as large as $50 \%$. More recently, enhanced spin injection was reported in amine-ended molecular junctions via mechanical strain which was explained by pronounced spin-up transmission due to hybridization between the $d_{y z}$ and $\pi$ orbitals of a molecule [14]. 
In the present paper, we propose theoretically a mechanism for tuning the spin-filtering ratio in molecular junctions based on clear symmetry arguments and quantum interference effects. In particular, a mechanical strain can be used to modify the nanojunction symmetry switching on or off new conduction channels. The idea is illustrated for two possible examples: a benzene molecule (perpendicular vs tilted configuration) and a short Si chain (zigzag vs linear configuration) connecting two ferromagnetic Ni electrodes. We find, in particular, that in both cases the majority-spin conductance is strongly quenched in low-symmetry configurations (a tilted benzene or a zigzag Si chain) as a consequence of destructive quantum interference. The minority-spin conductance is, on the contrary, significantly enhanced due to stronger hybridization of molecular orbitals with ferromagnetic electrodes. These opposite trends for two spins lead to strong modulation of resulting spin polarization of conductance as a function of strain.

It should be noted that previous studies on quantum interference in electron transport focused on different geometrical conformations or connections of molecules to nonmagnetic electrodes [15-20]. The well-known effect is, for example, the destructive quantum interference occurring when the benzene molecule is connected to electrodes in a meta or ortho configuration, in contrast to the para orientation [15,16,21]. Very recently, the destructive $\sigma$ interference was also reported in nonmagnetic molecular junctions, resulting in complete suppression of the transmission close to the Fermi level [22]. Our results demonstrate how the quantum interference can be exploited for strong spin polarization of the electric current in molecular junctions with ferromagnetic electrodes. We show, moreover, that the destructive interference (blocking fully one spin channel) can be easily tuned (switched on or off) by a mechanical strain.

\section{RESULTS AND DISCUSSION}

The spin-polarized electronic structure calculations were performed using the QUANTUM ESSPRESSO (QE) [23] package based on the density functional theory (DFT). We used the Perdew-Burke-Ernzerhof [24] exchange-correlation functional with ultrasoft pseudopotentials to describe electron-ion interactions. For electron transport two codes have been used. Spin-polarized conductances (given by electron transmission at the Fermi energy) for different molecular junctions shown in Fig. 1 were evaluated using the plane-wave scatteringbased approach as implemented in the PWCOND code [25]. All the energy-dependent transmissions presented in the other figures were calculated using a homemade tight-binding (TB) code [26]. TB parameters, including overlap matrices, on-site energies, and hopping integrals, were extracted from $a b$ initio QE calculations by projecting the self-consistent Hamiltonian onto the basis of atomic wave functions provided by pseudopotential files. This procedure is rather similar to the one used for calculating the projected density of states (PDOS). The standard nonequilibrium Green's function formalism was then employed to obtain the transmission function:

$$
T(E)=\operatorname{Tr}\left[\Gamma_{L}(E) G^{r}(E) \Gamma_{R}(E) G^{a}(E)\right]
$$
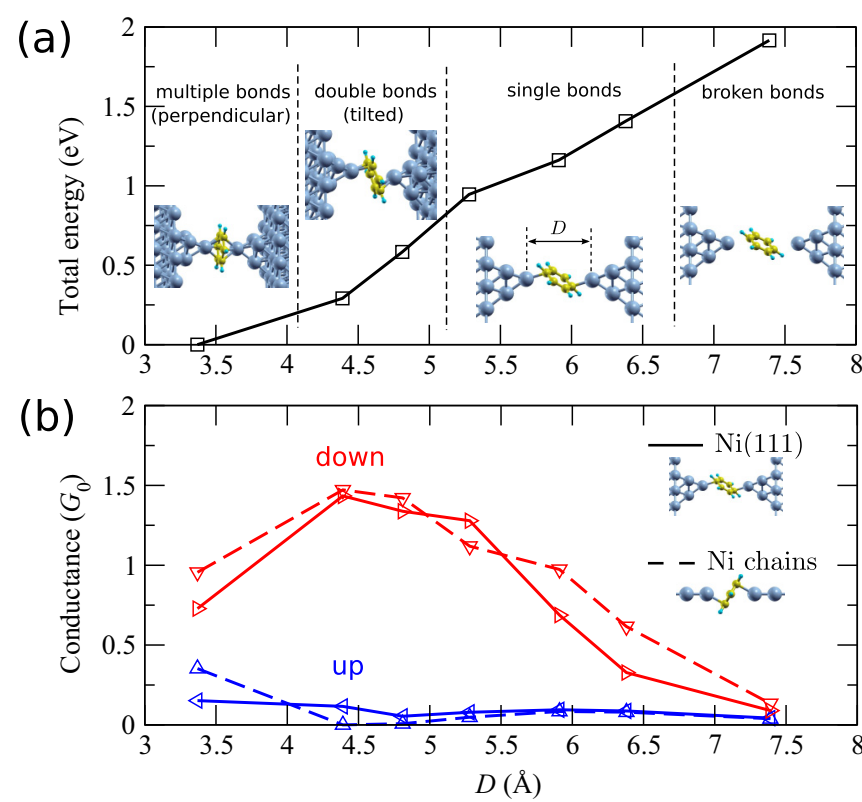

FIG. 1. DFT calculations for Ni/benzene/Ni junctions. (a) Total energy as a function of electrode-electrode separation $D$ (the first point was set as zero). The insets show the representative relaxed geometries at different values of $D$. (b) Spin-dependent conductance (given by the Fermi energy transmission) as a function of stretching for both crystalline $\mathrm{Ni}(111)$ electrodes (solid lines) and model semiinfinite Ni chains (dashed lines).

where $G^{r, a}$ are the retarded and advanced Green's functions of the central region (including the molecule and some portions of left and right electrodes) and $\Gamma_{L, R}$ are the coupling matrices of the central region to the left and right electrodes, respectively. For spin-polarized calculations all the quantities also depend on the spin index, but two spin channels are not coupled (without spin-orbit interactions and for collinear magnetism).

\section{A. Strain-dependent spin filtering in $\mathrm{Ni}$ /benzene junctions}

We start our discussion with $\mathrm{Ni} /$ benzene/Ni molecular junctions, shown in Fig. 1, which were recently studied extensively with respect to large anisotropic magnetoresistance [27]. To determine possible geometries we gradually stretch the junctions up to the breaking point, starting from the benzene molecule, which is perpendicular to the direction of the current flow. The molecular contact is described in a supercell containing the benzene molecule and two four-atom $\mathrm{Ni}$ pyramids attached to a $\mathrm{Ni}(111)$ slab containing five and four atomic layers on the left and right sides, respectively (see the insets of Fig. 1). During the stretching process, we move apart the two electrodes stepwise and relax all the atomic positions except for two bottom Ni layers on both sides (which were kept in their bulklike positions) until the junction was broken.

As presented in Fig. 1(a), our ab initio structural optimization shows that upon increasing the electrode-electrode separation $D$ the benzene molecule is connected to $\mathrm{Ni}$ electrodes in three different ways before the breaking point: (i) by all six $\mathrm{C}$ atoms bound symmetrically to $\mathrm{Ni}$ apex atoms 
(direct $\pi$-bound perpendicular orientation for small separations), (ii) by two $\mathrm{C}$ atoms on each side (mixed $\pi$ and $\sigma$-bound tilted orientation for intermediate separations), and (iii) by only one $\mathrm{C}$ atom on each side (larger separations). These results are similar to those reported previously for Pt/benzene/Pt nanojunctions [28,29]. Very recently, Rakhmilevitch et al. [27] successfully created single-molecule junctions based on $\mathrm{Ni}$ electrodes and benzene molecules using the mechanically controlled break junction (MCBJ) technique, which supports our theoretical results. Thus, during the stretching, the symmetry of the molecule connection is lowered from the highly symmetric configuration (i) to lowsymmetry geometries (ii) and (iii).

The spin-polarized conductance as a function of stretching is shown in Fig. 1(b). The results for two types of electrodes are presented: realistic $\mathrm{Ni}(111)$ electrodes (solid lines) which were used for atomic relaxations of Fig. 1(a) and model $\mathrm{Ni}$ chain electrodes. In both cases the conductance is found to be highly strain dependent in both spin channels. One can distinguish three regimes clearly correlating with the three benzene orientations. In the perpendicular configuration (i) the conductance is rather low and moderately spin polarized. In the tilted orientation (ii) the spin-down conductance is strongly enhanced, while the spin-up conductance is, in contrast, suppressed, which gives rise to a dramatic increase in the spin polarization of the conductance. Very interestingly, the suppression of the spin-up conductance is complete for model Ni electrodes, a point which will be addressed in detail in the following. In the single-bond regime (iii) the spin-down (spin-up) conductance starts to decrease (increase), reducing again the spin-filtering ratio.

\section{B. Ni chain/benzene junctions: Interference effects}

With the aim to understand a dramatic change in the spinpolarized conductance between orientations (i) and (ii), we consider first a model case with $\mathrm{Ni}$ chain electrodes, as shown at the top of Fig. 2. We plot in Fig. 2(a) the spin-resolved transmission function for three configurations, namely, perpendicular and tilted with $\theta=20^{\circ}$ and $30^{\circ}$ configurations. All three geometries have the same separation distance of $D=4.39 \AA$ taken from the most representative double-bond configuration (see Fig. 1). In order to understand electron transport better, we also plot in Figs. 2(b) and 2(c) the density of states (DOS) projected onto highest occupied molecular orbitals (HOMOs) and lowest unoccupied molecular orbitals (LUMOs), for spin-up polarization. It is known (see, e.g., Ref. [30]) that the Ni atomic chain has only one $s$ band around the Fermi energy for the spin-up channel, while five more $d$ bands are also available for the spin-down one.

We first analyze the spin-up channel. Clearly, its conductance (determined by transmission at the Fermi energy) gets significantly suppressed when the molecule is tilted. Interestingly, this effect can be interpreted as a result of destructive quantum interference. In the perpendicular configuration, the frontier orbitals (twofold-degenerate LUMO and HOMO) are all strictly orthogonal to the $\mathrm{Ni} s$ channel [see the insets in Fig. 2(c)] and do not, therefore, manifest in transport at energies from -0.5 to $2.7 \mathrm{eV}$ with respect to the Fermi level, where only the $s$ channel is present in

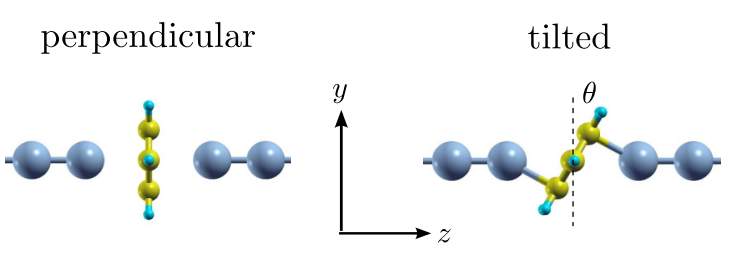

(a)

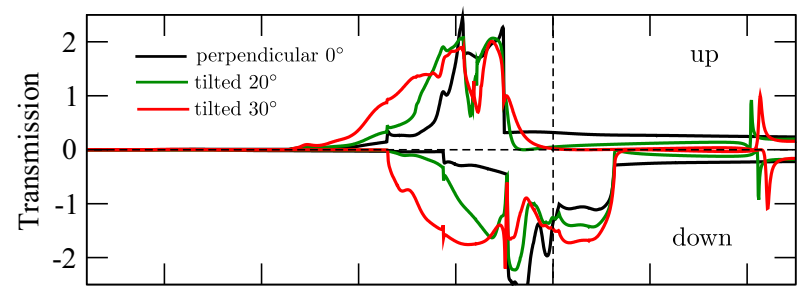

(b)

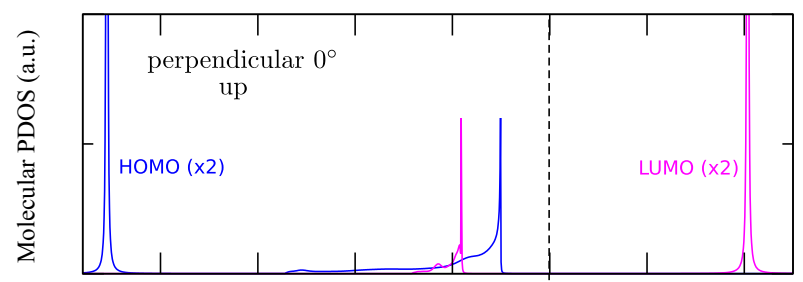

(c)

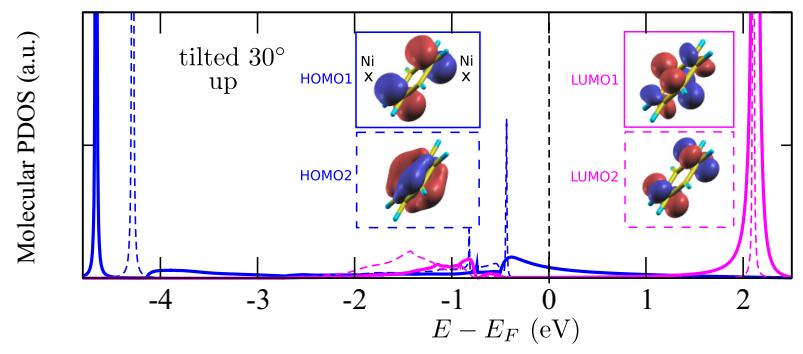

FIG. 2. TB transport calculations of model benzene junctions with $\mathrm{Ni}$ atomic chains as electrodes for different benzene tilting angles $\theta$. (a) Spin-resolved transmissions as a function of $\theta$. Due to destructive interference from HOMO1 and LUMO1 orbitals, activated by tilting, the spin-up transmission gets fully suppressed at the Fermi energy. (b) DOS projected onto HOMO and LUMO molecular orbitals for the spin-up channel in the perpendicular configuration. (c) The same for the tilted configuration of $\theta=30^{\circ}$. Wave functions of all the HOMO and LUMO orbitals are shown in insets where the positions of contact Ni chain atoms are also indicated. Only HOMO1 (solid blue line) and LUMO1 (solid pink line), due to their symmetry (even with respect to the $Y Z$ plane), have nonzero overlap with the Ni $s$ channel.

Ni chains. Small, nonzero transmission here is attributed to the HOMO-2 orbital which is rotationally symmetric (see the Supplemental Material [31], Fig. S1) and has thus a perfect overlap with $\mathrm{Ni} s$ states, providing a rather constant tunneling transmission of about $0.3 G_{0}$ at the Fermi level. For tilted orientation only one symmetry plane, $Y Z$, is left. Two orbitals, HOMO1 and LUMO1, which are both symmetric with respect to that plane [see the insets in Fig. 2(c)], can now overlap with the (symmetric) $s$ channel of $\mathrm{Ni}$, while two others, HOMO2 and LUMO2, are antisymmetric and therefore remain decoupled. This can be seen from the DOS analysis shown in Fig. 2(c): the twofold-degenerate HOMO splits into two peaks located at about $-4.7 \mathrm{eV}$ (HOMO1) and 


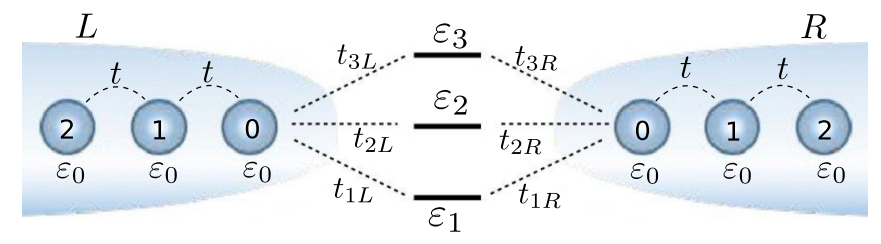

(i) only level 1

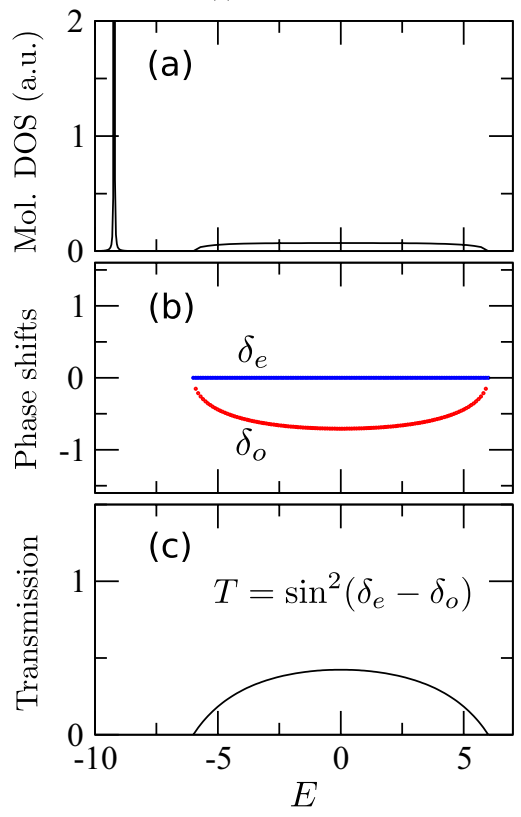

(ii) different symmetry of 2 and 3

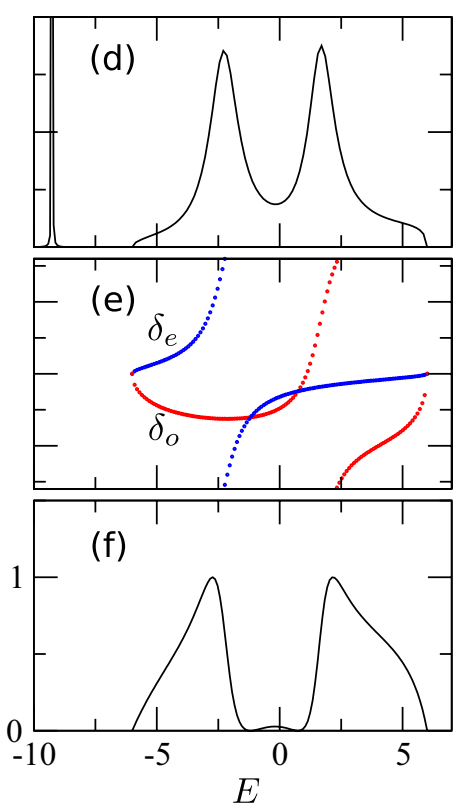

(iii) same symmetry of 2 and 3
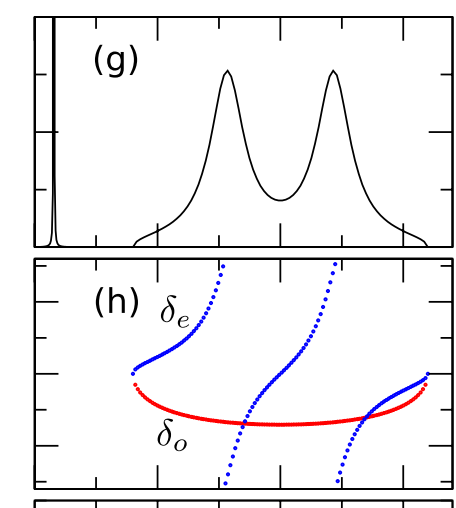

(i)

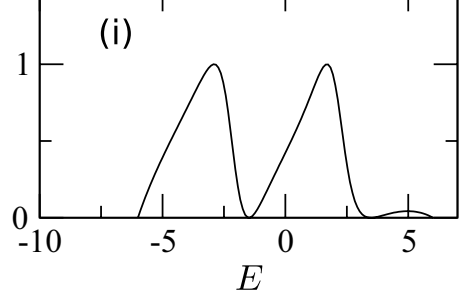

FIG. 3. Simple three-level 1D model providing different quantum interference patterns in transmission. Molecular (summed over all three levels) DOS, even/odd phase shifts, and transmission functions derived from them are shown in the top, middle, and bottom panels, respectively. Three different cases are considered: (i) only the first level, $\varepsilon_{1}=-7$, is coupled to chains with $t_{1 L}=-t_{1 R}=3$ [(a)-(c)], (ii) levels 2 and $3, \varepsilon_{2}=-2, \varepsilon_{3}=2$, are also added, the first coupled symmetrically $\left(t_{2 L}=t_{2 R}=1\right)$ and the second one coupled antisymmetrically $\left[t_{3 L}=-t_{3 R}=1\right.$; (d)-(f)], and (iii) levels 2 and 3 are both coupled symmetrically, $t_{2 L}=t_{2 R}=t_{3 L}=t_{3 R}=1$ [(g)-(i)]. The electrode conduction band spreads within the energy interval $[-6 ; 6]$, which corresponds to $\varepsilon_{0}=0, t=3$, and the Fermi level is set to zero.

$-4.3 \mathrm{eV}$ (HOMO2), and the finite DOS at [ $-0.4 \mathrm{eV} ; 1.0 \mathrm{eV}]$ of HOMO1 is due to finite hybridization with $s$ states of Ni. Similarly, the two LUMOs, located at about $2 \mathrm{eV}$, show up as a sharp ( $\delta$-like) peak (LUMO2) and a rather broad resonance (LUMO1). These additional conduction paths provided by HOMO1 and LUMO1 interfere with the former tunneling one, resulting in a Fano-like shape of spin-up transmission and its strong suppression in a broad energy window of $[-0.4 \mathrm{eV}$; $2.0 \mathrm{eV}]$.

For spin-down polarization, the conductance is dominated by additional $d$ channels. In particular, two $d_{x z, y z} \mathrm{Ni}$ bands appear at $E<0.7 \mathrm{eV}$, increasing significantly the spin-down transmission. Those bands have nonzero overlap with HOMOs even in the perpendicular configuration and hybridize also to LUMO states when the molecule is tilted. The stronger hybridization of spin-down $d_{x z, y z}$ bands to frontier orbitals is therefore responsible for the increase in transmission at $E<0.7 \mathrm{eV}$ when the molecule is tilted.

It should be noted here that more sophisticated transport calculations with PWCOND have produced very similar transmission curves (see the Supplemental Material [31], Fig. S2), reproducing very well the same features, in particular, the destructive interference pattern in the spin-up channel. This validates and supports our TB calculations, which allow us to explore in more detail our findings and the mechanism behind them.

\section{Interference from scattering phase shifts}

The actual shape of spin-up transmission around the Fermi energy resulting from the interference effects mentioned above can be well rationalized in terms of so-called scattering phase shifts (see, e.g., Ref. [32]). We illustrate the idea on a simple three-level TB model, as shown in Fig. 3, with the Hamiltonian

$$
\begin{aligned}
& \sum_{\alpha \in L, R}\left[\varepsilon_{0} \hat{c}_{\alpha}^{\dagger} \hat{c}_{\alpha}+\left(t \hat{c}_{\alpha}^{\dagger} \hat{c}_{\alpha+1}+\text { H.c. }\right)\right] \\
& +\sum_{i=1}^{3}\left[\varepsilon_{i} \hat{c}_{i}^{\dagger} \hat{c}_{i}+\left(t_{i L} \hat{c}_{i}^{\dagger} \hat{c}_{L 0}+t_{i R} \hat{c}_{i}^{\dagger} \hat{c}_{R 0}+\text { H.c. }\right)\right],
\end{aligned}
$$

where the first term stands for the left and right semi-infinite chains with a single band, simulating the spin-up $s$ band of a $\mathrm{Ni}$ chain. The second term describes the three levels coupled to apex atoms (numbered as 0 ) of the chains. Here, the first level is placed at -7 (in arbitrary energy units), simulating the important HOMO-2 benzene orbital contributing to the 
perpendicular
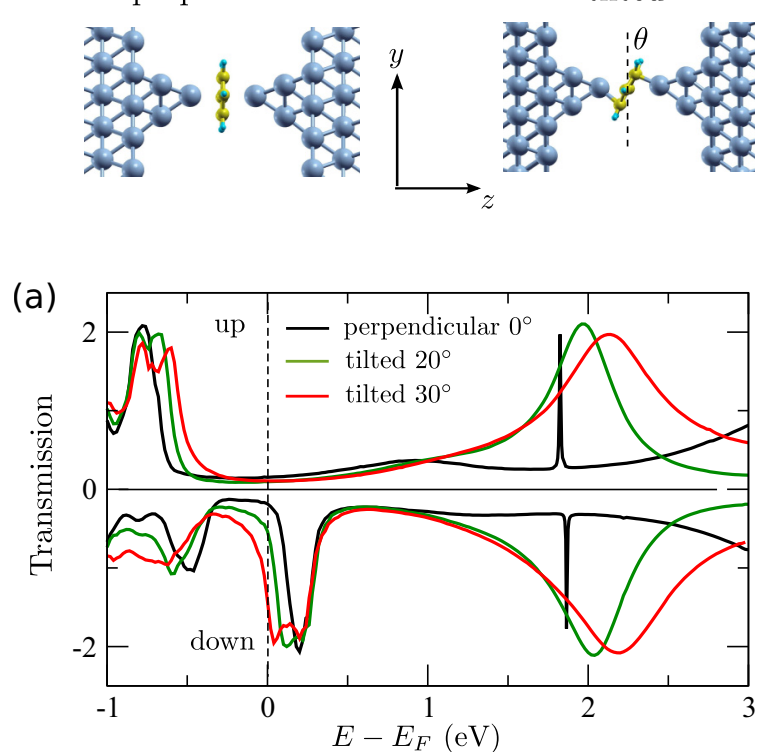

(b)

(c)

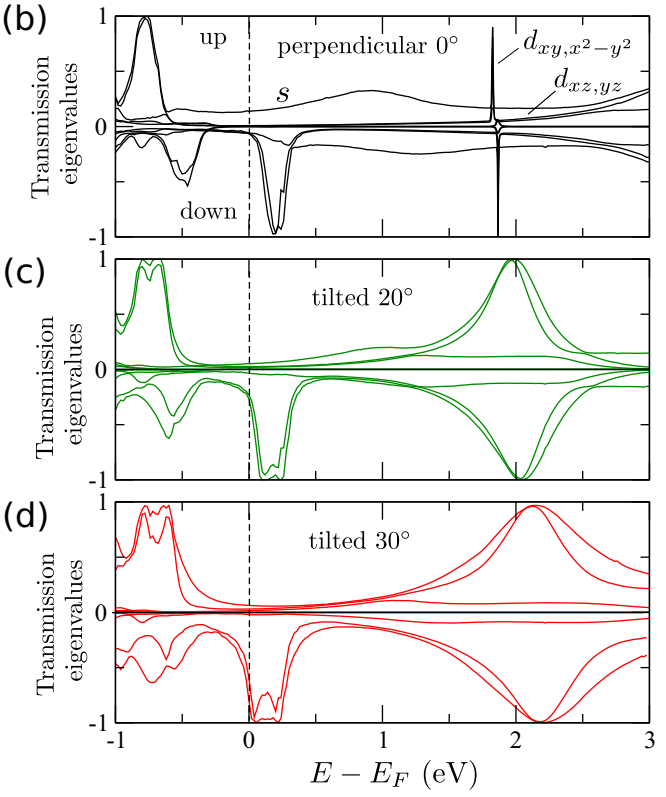

FIG. 4. TB calculations for benzene junctions with realistic fcc $\mathrm{Ni}(111)$ electrodes: (a) spin-resolved transmissions as a function of tilting angle $\theta$ and (b)-(d) transmission eigenchannels for perpendicular and tilted configurations with $\theta=20^{\circ}$ and $30^{\circ}$.

tunneling transmission at the Fermi energy (set to zero). Two other levels, set at -2 and 2, simulate HOMO1 and LUMO1, respectively, which could couple to the chains in the tilted benzene configuration. For this single-band case the transmission probability can be expressed [32] as $T=\sin ^{2}\left(\delta_{e}-\delta_{o}\right)$, where $\delta_{e / o}$ are phase shifts in even and odd combinations of two electron waves coming from the left and from the right chains caused by coupling to the molecule (levels). The reference system (zero phase shifts) is the one in which the two chains are fully disconnected (no molecule). From the other side, by the Friedel sum rule, $d \delta_{e / o}(E) / d E=\pi \Delta \rho_{e / o}(E)$, where $\Delta \rho_{e / o}$ are additional DOSs of even or odd symmetry (with respect to the transport direction) due to the molecule.

Figures 3(a)-3(c) represent the case where only level 1 is coupled to the chains, which would correspond to the perpendicular benzene orientation. Due to the $\pi$ character of HOMO-2 (which is also true for HOMOs and LUMOs, see the Supplemental Material [31], Fig. S1) its coupling to the left and right chains is antisymmetric, leading to a small (odd) DOS around the Fermi energy [Fig. 3(a)]. That generates rather constant $\delta_{o}$ [Fig. 3(b)], which results in a small tunneling transmission around zero energy, as seen in Fig. 3(c). The case of tilted orientation is simulated in Figs. 3(d)-3(f) when two other levels are also included. Their coupling to the chains should be symmetric (level 2) and antisymmetric (level 3), as can be clearly inferred from HOMO1 and LUMO1 shown in Fig. 2(c). That will result in a rapid increase of $\delta_{e}$ and then of $\delta_{o}$, turning the transmission exactly zero twice on both sides of the Fermi energy where $\delta_{o}=\delta_{e}$, which agrees clearly with our previous calculations [Fig. 2(a)]. Very interestingly, the efficient suppression of transmission around the Fermi energy is only achieved if levels 2 and 3 (HOMO1 and LUMO1) have different even and odd symmetries, like in the discussed case. If they had the same symmetry, as presented in Figs. 3(g)-3(i) for symmetric couplings, the transmission would drop to zero always to the right of either HOMO1 or LUMO1 peaks, re- maining finite in between. Note that, unlike transmissions, the molecular DOS shows a very similar (trivial) shape for both types of couplings [Figs. 3(d) and 3(g)]: two Lorentzian-like peaks from levels 2 (HOMO1) and 3 (LUMO1) superposed on a smooth feature from level 1 (HOMO-2).

\section{D. $\mathrm{Ni}(111) /$ benzene junctions}

Having understood the main idea in simple model systems, we pass now to more realistic nanojunctions by replacing semi-infinite $\mathrm{Ni}$ wires with fcc $\mathrm{Ni}(111)$ electrodes, as shown in Fig. 4. Here, we show three different geometries as in the case of the model systems discussed above. Figure 4(a) presents the spin-resolved transmission for those configurations. Clearly, spin-down conductance is again significantly enhanced when the molecule is tilted due to broadening of the transmission peak centered at around $E=0.2 \mathrm{eV}$. This peak is due to the offset of extra $d_{x z, y z} \mathrm{Ni}$ states, and its broadening reflects stronger hybridization with molecular states (mainly with HOMO) in the low-symmetry tilted configuration, similar to the case of model Ni chains.

The situation for spin-up polarization is more complex: the conductance is only slightly reduced in tilted geometries, and the transmission curve now does not show clear signatures of interference (points of zero transmission). In order to get a better understanding we plot in Figs. 4(b)-4(d) spin-up transmission eigenvalues for all configurations. As discussed in the previous section, spin-up transmission with $\mathrm{Ni}$ chains was essentially provided by a single $s$ channel in a broad energy window [ $-0.5 \mathrm{eV} ; 2.7 \mathrm{eV}]$. On the contrary, as can be learned from the perpendicular configuration in Fig. 4(b), five conduction channels are available for transport with realistic Ni electrodes. Those channels are mainly provided by $s, d_{x z, y z}$, and $d_{x y, x^{2}-y^{2}}$ orbitals of $\mathrm{Ni}$ apex atoms, so we label them accordingly in Fig. 4(b). Two LUMOs can couple only by symmetry (see the Supplemental Material [31], Fig. S1) to 
$d_{x y, x^{2}-y^{2}}$ channels and give rise to sharp (because of the weak coupling) peaks in transmission at about $1.8 \mathrm{eV}$. The other three channels are orthogonal to the LUMOs and are therefore in the off-resonant regime. A rather constant eigenvalue, of about 0.2 around the Fermi energy, is provided by the $s$ channel, which clearly dominates in the large energy window. Note that its transmission at the Fermi energy is slightly lower than the one for the model Ni chain case, about 0.35 [see Fig. 2(a)], reflecting the smaller $s$-orbital DOS at the Ni apex atoms (not shown). Finally, two degenerate $d_{x z, y z}$ channels show a small transmission of about 0.05 at the LUMO position, slowly increasing with energy.

When the molecule is tilted, due to symmetry reduction, LUMO1 (symmetric with respect to the $Y Z$ plane) starts to hybridize with the (symmetric) $s$ channel and also, much more strongly, with the (symmetric) $d_{y z}$ one, as can be clearly seen for the tilting angle of $20^{\circ}$ [Fig. 4(c)]. That results in a much more pronounced broadening of the LUMO1 orbital compared to the model $\mathrm{Ni}$ chain case. Moreover, two anticrossing points at about 1.3 and $2.3 \mathrm{eV}$ appear between the two symmetric channels. Interestingly, the transmission of the $s$ channel at the Fermi energy is again suppressed with respect to the one in the perpendicular configuration. In addition, the (antisymmetric) LUMO2 couples strongly to the (antisymmetric) $d_{x z}$ channel, giving rise to another broad transmission feature at around $2 \mathrm{eV}$. We note that the two other channels, with $d_{x y, x^{2}-y^{2}}$ symmetry, almost do not contribute to the transport at the energies of interest, from -0.5 to $3 \mathrm{eV}$. When the molecule is further tilted, the hybridization of both LUMOs (mainly to $d_{x z, y z} \mathrm{Ni}$ states) is increased, as can be seen for a tilting angle of $30^{\circ}$ [Fig. 4(d)]. Finally, we note that in all the discussed cases (Figs. 2 and 4) the electrode-electrode separation was kept fixed, $D=$ $4.39 \AA$, in order to analyze clearly the effect of molecule tilting. The effect of increasing $D$ on spin polarization was found to be much smaller, leading to a trivial decrease in both spin-up and -down conductances (see the Supplemental Material [31], Fig. S3). We can conclude therefore that by tilting the molecule the $s$-channel contribution to the spin-up conductance is suppressed due to quantum interference, but the overall effect is somewhat hindered because of additional $d$ channels appearing in the case of realistic Ni electrodes.

\section{E. Three-atom Si junctions with Ni chain electrodes}

The symmetry arguments to control the spin-filtering property discussed so far are, in fact, quite general and can be realized in some other situations. We have found, for example, that similar interference patterns in the transmission function can appear for some atomic chains (see Fig. 5, top panel) during the transition from linear to zigzag geometry. Such chains can be realized in many possible ways, for example, out of various kinds of alkane or alkene molecules [33]. To demonstrate once again our principle we have chosen a simple model three-atom $\mathrm{Si}$ chain which could be stretched from a zigzag geometry (energetically more preferable) to an almost linear configuration before being broken. In the linear configuration, the twofold-degenerate HOMO and LUMO (originating from $p_{x}$ and $p_{y} \mathrm{Si}$ orbitals) are again strictly orthogonal by symmetry to the $s$-like Ni channel and are

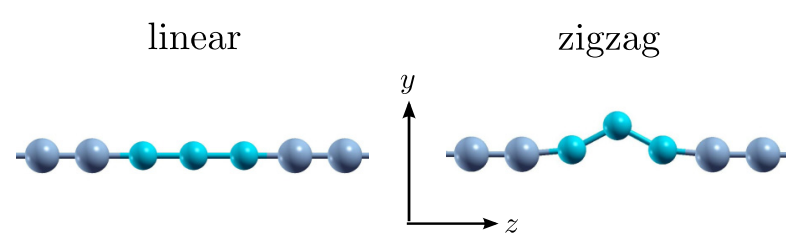

(a)

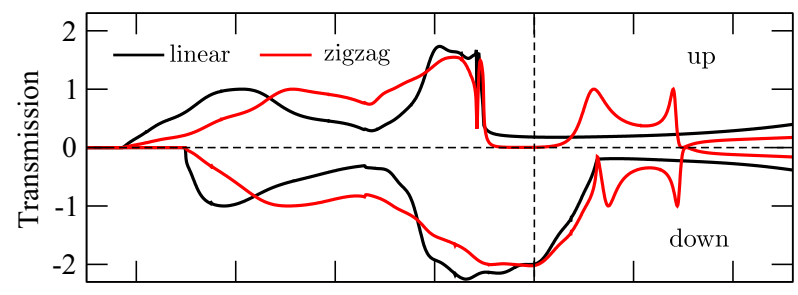

(b)

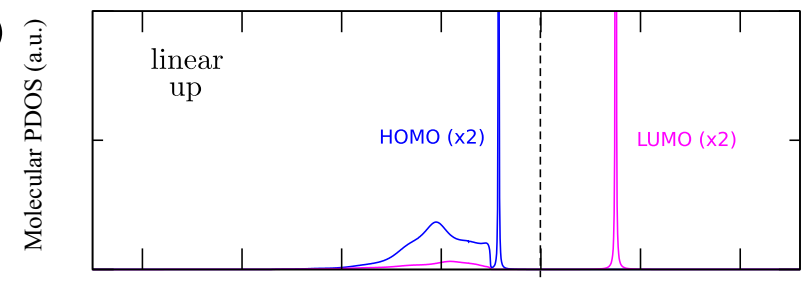

(c)

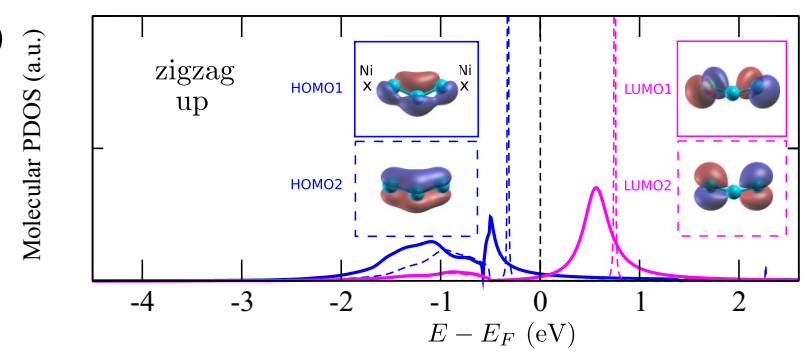

FIG. 5. TB transport calculations with model Ni chain electrodes for a three-atom Si chain in linear and zigzag geometries. (a) Spinresolved transmissions for two Si chain geometries. Due to destructive interference from HOMO1 and LUMO1, turned on in the zigzag geometry, the spin-up transmission gets fully suppressed at the Fermi energy. (b) PDOS on the Si chain for the spin-up channel in the linear configuration. (c) The same for the zigzag configuration. Wave functions of all the HOMOs and LUMOs are shown in the insets. Only HOMO1 (solid blue line) and LUMO1 (solid pink line), due to their symmetry (even with respect to the $Y Z$ plane), could overlap with a Ni $s$ channel.

therefore fully transparent for electron transport around the Fermi energy, which is dominated by tunneling through other $p_{z}$-character $\mathrm{Si}$ orbitals (not shown). In the zigzag configuration, due to the lack of full axial symmetry, HOMO1 and LUMO1 start to overlap and to interfere destructively with the background tunneling $s$ channel (all states being symmetric in the $Y Z$ mirror plane). HOMO2 and LUMO2, being antisymmetric, remain orthogonal to the $s$ channel and appear as $\delta$-like peaks in the molecular DOS at about -0.3 and $0.8 \mathrm{eV}$, respectively. This results again in complete suppression of spin-up transmission around the Fermi energy and to the full spin polarization of electric current in the zigzag geometry. Note that HOMO1 and LUMO1 have again different even and odd symmetries with respect to the $X Y$ plane perpendicular to the transport direction and passing through the central 
$\mathrm{Si}$ atom, the important condition to suppress efficiently the transmission in the HOMO-LUMO gap region.

\section{CONCLUSIONS}

We have suggested a possible way to manipulate the spinfiltering property of single-molecule junctions based on clear symmetry arguments. The important point is the change in nanojunction symmetry by, for example, a mechanical strain, which can switch on and off new channels for electron propagation (via molecular orbitals which were before inactive) and thus induce new interference patterns in the electron transmission function around the Fermi energy. Using two representative systems, a benzene molecule and three-atom $\mathrm{Si}$ chains bridging two Ni electrodes, we have shown that when the symmetry is lowered (a tilted benzene molecule or zigzag $\mathrm{Si}$ chain), the spin-up conductance gets strongly suppressed due to destructive interference of the main $s$ channel with (frontier) HOMOs and LUMOs. It was demonstrated, moreover, that those orbitals must have different (even and odd) symmetries with respect to the transport direction in order to provide strong suppression of spin-up transmission, which is the case for both molecular junctions. The spin-down conductance is, in contrast, enhanced due to stronger molecule-metal hybridization. Suggested symmetry arguments were shown to work perfectly for model systems with $\mathrm{Ni}$ chain electrodes, giving fully spin polarized conductance in low-symmetry configurations. For realistic Ni electrodes, the destructive interference in the spin-up channel appears somewhat hidden due to the multichannel character of the electron transport, in particular, due to the presence of two symmetric channels (of $s$ and $d_{y z}$ character), to which both the symmetric HOMO1 and LUMO1 can couple in the low-symmetry configuration. Finally, it should be noted that the ratio of spin-filtering modulation depends on a background value of the (s-channel) spin-up conductance (to be suppressed), which was rather small in our case but could probably be found to be much larger for some other systems. We believe that our findings will be important not only from the conceptual point of view but also for exploring novel functionalities for spin-based devices involving solid symmetry arguments and quantum interference effects.

\section{ACKNOWLEDGMENTS}

This work was performed using HPC computation resources from TGCC-GENCI (Grant No. A0040910407). D.L. was supported by the Alexander von Humboldt Foundation through a Fellowship for Postdoctoral Researchers.
[1] A. R. Rocha, V. M. Garcia-Suarez, S. W. Bailey, C. J. Lambert, J. Ferrer, and S. Sanvito, Nat. Mater. 4, 335 (2005).

[2] S. Sanvito, Nat. Phys. 60, 562 (2010).

[3] The spin polarization is defined as $\mathrm{SP}=\left(G_{\uparrow}-G_{\downarrow}\right) /\left(G_{\uparrow}+\right.$ $G_{\downarrow}$ ), where $G_{\uparrow}$ and $G_{\downarrow}$ are the conductances of the majorityand minority-spin channels, respectively.

[4] D. Çakır, D. M. Otálvaro, and G. Brocks, Phys. Rev. B 90, 245404 (2014).

[5] S. Schmaus, A. Bagrets, Y. Nahas, T. K. Yamada, A. Bork, M. Bowen, E. Beaurepaire, F. Evers, and W. Wulfhekel, Nat. Nanotechnol. 6, 185 (2011).

[6] A. Bagrets, S. Schmaus, A. Jaafar, D. Kramczynski, T. K. Yamada, M. Alouani, W. Wulfhekel, and F. Evers, Nano Lett. 12, 5131 (2012).

[7] N. M. Caffrey, P. Ferriani, S. Marocchi, and S. Heinze, Phys. Rev. B 88, 155403 (2013).

[8] N. Atodiresei, J. Brede, P. Lazić, V. Caciuc, G. Hoffmann, R. Wiesendanger, and S. Blügel, Phys. Rev. Lett. 105, 066601 (2010).

[9] S. L. Kawahara, J. Lagoute, V. Repain, C. Chacon, Y. Girard, S. Rousset, A. Smogunov, and C. Barreteau, Nano Lett. 12, 4558 (2012).

[10] D. Li, C. Barreteau, S. L. Kawahara, J. Lagoute, C. Chacon, Y. Girard, S. Rousset, V. Repain, and A. Smogunov, Phys. Rev. B 93, 085425 (2016).

[11] I. N. Sivkov, O. O. Brovko, D. I. Bazhanov, and V. S. Stepanyuk, Phys. Rev. B 89, 075436 (2014).

[12] D. Jacob, J. Fernández-Rossier, and J. J. Palacios, Phys. Rev. B 74, 081402 (2006).

[13] A. Bagrets, N. Papanikolaou, and I. Mertig, Phys. Rev. B 70, 064410 (2004).
[14] Y. H. Tang and C. J. Lin, J. Phys. Chem. C 120, 692 (2016).

[15] T. Hansen, G. C. Solomon, D. Q. Andrews, and M. A. Ratner, J. Chem. Phys. 131, 194704 (2009).

[16] G. C. Solomon, D. Q. Andrews, R. H. Goldsmith, T. Hansen, M. R. Wasielewski, R. P. Van Duyne, and M. A. Ratner, J. Am. Chem. Soc. 130, 17301 (2008).

[17] S. V. Aradhya, J. S. Meisner, M. Krikorian, S. Ahn, R. Parameswaran, M. L. Steigerwald, C. Nuckolls, and L. Venkataraman, Nano Lett. 12, 1643 (2012).

[18] P. Sam-ang and M. G. Reuter, New J. Phys. 19, 053002 (2017).

[19] A. Borges, J. Xia, S. H. Liu, L. Venkataraman, and G. C. Solomon, Nano Lett. 17, 4436 (2017).

[20] B. Huang, X. Liu, Y. Yuan, Z.-W. Hong, J.-F. Zheng, L.-Q. Pei, Y. Shao, J.-F. Li, X.-S. Zhou, J.-Z. Chen et al., J. Am. Chem. Soc. 140, 17685 (2018).

[21] R. Miao, H. Xu, M. Skripnik, L. Cui, K. Wang, K. G. L. Pedersen, M. Leijnse, F. Pauly, K. Wärnmark, E. Meyhofer et al., Nano Lett. 18, 5666 (2018).

[22] M. H. Garner, H. Li, Y. Chen, T. A. Su, Z. Shangguan, D. W. Paley, T. Liu, F. Ng, H. Li, S. Xiao et al., Nature 558, 415 (2018).

[23] P. Giannozzi, S. Baroni, N. Bonini, M. Calandra, R. Car, C. Cavazzoni, D. Ceresoli, G. L. Chiarotti, M. Cococcioni, I. Dabo et al., J. Phys.: Condens. Matter 21, 395502 (2009).

[24] J. P. Perdew, K. Burke, and M. Ernzerhof, Phys. Rev. Lett. 77, 3865 (1996).

[25] A. Smogunov, A. Dal Corso, and E. Tosatti, Phys. Rev. B 70, 045417 (2004).

[26] G. Autès, C. Barreteau, D. Spanjaard, and M.-C. Desjonquères, Phys. Rev. B 77, 155437 (2008). 
[27] D. Rakhmilevitch, S. Sarkar, O. Bitton, L. Kronik, and O. Tal, Nano Lett. 16, 1741 (2016).

[28] M. Kiguchi, O. Tal, S. Wohlthat, F. Pauly, M. Krieger, D. Djukic, J. C. Cuevas, and J. M. van Ruitenbeek, Phys. Rev. Lett. 101, 046801 (2008).

[29] T. Yelin, R. Vardimon, N. Kuritz, R. Korytár, A. Bagrets, F. Evers, L. Kronik, and O. Tal, Nano Lett. 13, 1956 (2013).

[30] A. Smogunov and Y. J. Dappe, Nano Lett. 15, 3552 (2015).
[31] See Supplemental Material at http://link.aps.org/supplemental/ 10.1103/PhysRevB.99.115403 for electronic structure of isolated Benzene molecule and additional transport calculations.

[32] P. P. Baruselli, M. Fabrizio, A. Smogunov, R. Requist, and E. Tosatti, Phys. Rev. B 88, 245426 (2013).

[33] T. A. Su, H. Li, R. S. Klausen, J. R. Widawsky, A. Batra, M. L. Steigerwald, L. Venkataraman, and C. Nuckolls, J. Am. Chem. Soc. 138, 7791 (2016). 